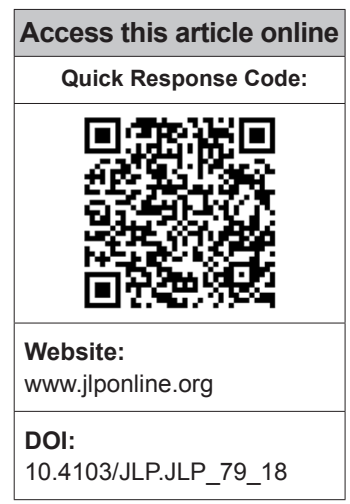

Department of Microbiology, Nizam's Institute of Medical Sciences, Hyderabad,

Telangana, India

Address for correspondence: Dr. Padmaja Kanne,

Department of Microbiology, Nizam's Institute of Medical Sciences, Panjagutta,

Hyderabad - 500 082,

Telangana, India E-mail: kannepadmaja@ gmail.com

Submission: 07-06-2018 Accepted: 24-06-2018

\title{
Extraintestinal infections caused by nontyphoidal Salmonella from a tertiary care center in India
}

\author{
Sukanya Sudhaharan, Padmaja Kanne, Lakshmi Vemu, Aparna Bhaskara
}

\begin{abstract}
:
BACKGROUND: Infection with Salmonella has become an increasing problem worldwide. Recently, nontyphoid Salmonella (NTS) has become a global concern causing threat to the health of human. It causes gastrointestinal infection which may be self-limiting, but invasive infections may be fatal, requiring appropriate therapy. This study was done to analyze the spectrum of NTS infections causing extraintestinal infections and its susceptibility pattern from a tertiary care center in India.
\end{abstract}

MATERIALS AND METHODS: The medical records of 27 patients whose cultures were positive for NTS between the years 2013-2016 were included in this retrospective study. The relevant demographic, clinical, and laboratory data were analyzed.

RESULTS: Among the 27 patients, predominant patients were in the age group of $20-30$ years. The male to female ratio is $1.7: 1$. Salmonella typhimurium was the predominant NTS isolated among $15 / 27$ (55.5\%), followed by Salmonella enteritidis 4/27 (14.8\%). 18/27 (66.6\%) of NTS were isolated from blood. Nalidixic acid was sensitive in 2/15 of $S$. typhimurium, 2/4 of $S$. enteritidis and 1/3 of Salmonella weltevreden, while others are nalidixic acid-resistant implying resistance to quinolones. They were sensitive to other antibiotics reported.

CONCLUSION: This study highlights the spectrum of NTS causing extraintestinal infections which is an emerging infection occurring mostly in immunosuppressed individuals. There should be a high degree of clinical suspicion which would help in the early diagnosis and management of patients.

Key words:

Extraintestinal, focal, immunocompromised, invasive, nontyphoid Salmonella

\section{Introduction}

Calmonella is an enteroinvasive bacterium Swhich on ingestion bypass gastric defenses, multiply, and penetrate the intestinal mucosa. ${ }^{[1]}$ Infection with Salmonella has become an increasing problem worldwide. Salmonella is an important human pathogen comprising different serotypes of medical importance which can lead to considerable morbidity and mortality. ${ }^{[2]}$ There are 2600 serovars of Salmonella which are divided into typhoidal and nontyphoidal Salmonella (NTS). ${ }^{[3]}$ They cause a wide range

This is an open access journal, and articles are distributed under the terms of the Creative Commons Attribution-NonCommercial-ShareAlike 4.0 License, which allows others to remix, tweak, and build upon the work non-commercially, as long as appropriate credit is given and the new creations are licensed under the identical terms.

For reprints contact: reprints@medknow.com of intestinal and extraintestinal infections. ${ }^{[4,5]}$ Recently, NTS has become a global concern causing threat to the health of human. It causes gastrointestinal infection which may be self-limiting, but invasive infections occurring in the extraintestinal sites such as blood, bone, and joints, meninges may be fatal, requiring appropriate therapy. ${ }^{[2,6]}$ The global burden of NTS is 3.4 million annually. ${ }^{[7]}$ There were few studies about NTS causing extraintestinal infections from India. ${ }^{[8]}$ The prevalence of NTS serotypes helps in epidemiological and treatment purposes. ${ }^{[9]}$ This study was done to analyze the spectrum of NTS infections causing extraintestinal infections and its susceptibility pattern from a tertiary care center in India.

How to cite this article: Sudhaharan $\mathrm{S}$, Kanne $\mathrm{P}$, Vemu L, Bhaskara A. Extraintestinal infections caused by nontyphoidal Salmonella from a tertiary care center in India. J Lab Physicians 2018;10:401-5. 


\section{Materials and Methods}

The medical records of 27 patients whose cultures were positive for NTS between the years 2013-2016 were included in this retrospective study. The relevant demographic, clinical, and laboratory data were analyzed. The samples included blood, urine, purulent aspirates and body fluids. Blood was collected in 2 sets of blood culture bottles within $24 \mathrm{~h}, 10 \mathrm{ml}$ each in standard and FAN plus aerobic blood culture bottles and incubated in $\mathrm{BacT} /$ AlerT three-dimensional continuous monitoring blood culture system. Positive blood cultures and clinical samples were subcultured on 5\% sheep blood agar and chromogenic agar (CPS ID, bioMerieux, Marcy l'Etoile, France) and incubated at $37^{\circ} \mathrm{C}$ for $48 \mathrm{~h}$. Identification and antimicrobial susceptibility of the colonies were done by the Vitek-2 compact system in the laboratory.

The antibiotics tested were ampicillin $10 \mu \mathrm{g}$, ceftriaxone $30 \mu \mathrm{g}$, ceftazidime $30 \mu \mathrm{g}$, cefepime $30, \mu \mathrm{g}$ piperacillin-tazobactum $100 / 10 \mu \mathrm{g}$, ciprofloxacin $5 \mu \mathrm{g}$, levofloxacin $5 \mu \mathrm{g}$, cotrimoxazole $1.25 / 23.75 \mu \mathrm{g}$, and nalidixic acid $30 \mu \mathrm{g}$ as per CLSI guidelines (2016). Isolates identified as Salmonella were sent to Central Research Institute, Kasauli, Himachal Pradesh, India, for serotyping.

\section{Results}

Among the 27 patients, predominant patients were in the age group of 20-30 years [Figure 1]. The male to female ratio is 1.7:1. Salmonella typhimurium was the predominant NTS isolated among 15/27 (55.5\%), followed by Salmonella enteritidis 4/27 (14.8\%), Salmonella lindenberg 3/27 (11.1\%), and Salmonella weltevreden $3 / 27(11.1 \%)$ [Table 1]. 18/27 (66.6\%) of NTS were isolated from blood. The other sites include urine, pleural fluid, synovial fluid, ascitic fluid, and pus [Table 2]. Nalidixic acid was sensitive in 2/15 of $S$. typhimurium, 2/4 of $S$. enteritidis, and $1 / 3$ of $S$. weltevreden, while others are nalidixic acid resistant implying resistance to quinolones. They were sensitive to other antibiotics reported. Most

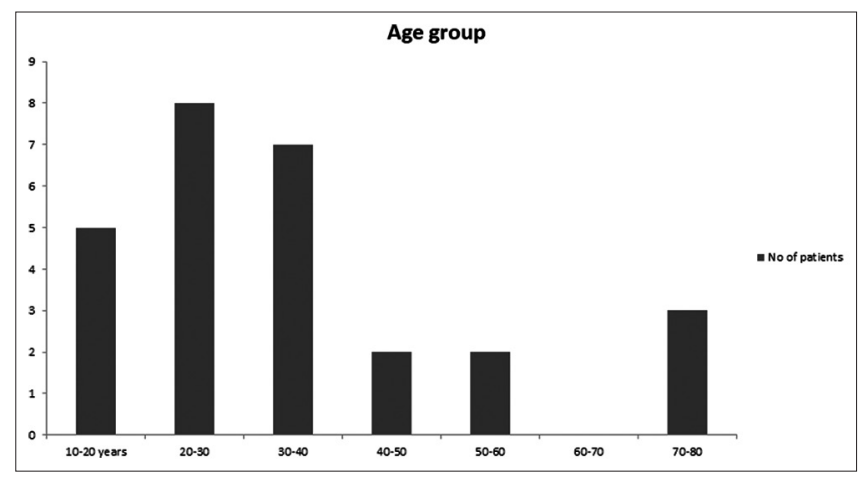

Figure 1: Demographic characteristics of nontyphoidal Salmonella of the patients were immunocompromised among which systemic lupus erythematosus (SLE) was the most common condition in $40.7 \%$ of the patients. The other risk factors were listed in Table 3.

\section{Discussion}

NTS is a zoonotic infection acquired from contaminated food of animal origin in industrialized countries. ${ }^{[7]}$ NTS is found in the gastrointestinal tract of animals and birds. It is transmitted to human beings through contaminated food and water with animal products and excreta. ${ }^{[9]}$

NTS is endemic in Sub-Saharan Africa causing bacteremia in children and adults, ${ }^{[5,10]}$ but the burden of NTS in India

Table 1: Total no of isolates $n=27$

\begin{tabular}{l|c|c|c}
\hline Organism & $\begin{array}{c}\text { Antigenic } \\
\text { formula }\end{array}$ & $\begin{array}{c}\text { No of } \\
\text { isolates }\end{array}$ & Percentage \\
\hline Salmonella typhimurium & $1,4,12: \mathrm{i}: 1,2$ & 15 & 55.5 \\
Salmonella enteritidis & $1,9,12: \mathrm{g}, \mathrm{m:}:$ & 4 & 14.8 \\
Salmonella lindenberg & $6,8: \mathrm{i}: 1,2$ & 3 & 11.1 \\
Salmonella weltevreden & $3,10: \mathrm{r}: \mathrm{z} 6$ & 3 & 11.1 \\
Salmonella kentucky & $8,20: \mathrm{i}: \mathrm{z} 6$ & 1 & 3.7 \\
Salmonella choleraesuis & $6,7: \mathrm{c}: 1,5$ & 1 & 3.7 \\
\hline
\end{tabular}

Table 2: Sample wise distribution of nontyphoid Salmonella

\begin{tabular}{llcc}
\hline Sample & Organism & $\begin{array}{c}\text { Number of Percentage } \\
\text { patients }\end{array}$ \\
\hline Blood $(n=18)$ & Salmonella typhimurium & 9 & 66.6 \\
& Salmonella enteritidis & 3 & \\
& Salmonella lindenberg & 3 & \\
& Salmonella weltevreden & 3 & \\
Synovial fluid & Salmonella typhimurium & 2 & 11.1 \\
$(n=3)$ & Salmonella kentucky & 1 & \\
Pus $(n=2)$ & Salmonella typhimurium & 1 & 7.4 \\
& Salmonella choleraesuis & 1 & \\
Urine $(n=2)$ & Salmonella typhimurium & 1 & 7.4 \\
& Salmonella enteritidis & 1 & \\
Pleural fluid $(n=1)$ & Salmonella typhimurium & 1 & 3.7 \\
Ascitic fluid $(n=1)$ & Salmonella typhimurium & 1 & 3.7 \\
\hline
\end{tabular}

Table 3: Risk factors in patients with nontyphoid Salmonella

\begin{tabular}{lc}
\hline Risk factor & $\begin{array}{c}\text { Number of } \\
\text { patients }\end{array}$ \\
\hline SLE & 11 \\
Haematological malignancy (ALL, AML) & 2 \\
Myeloproliferative disorder & 1 \\
Malignancy & 1 \\
Cirrhosis of liver & 2 \\
CKD & 1 \\
Postoperative (mitral valve replacement, Fracture femur) & 2 \\
PUO & 7 \\
\hline AML=Acute myeloid leukemia, ALL=Acute lymphoblastic leukemia, CKD=Chronic \\
kidney disease, SLE=Systemic lupus erythematosus, PUO=Pyrexia of unknown \\
origin
\end{tabular}


is not clear. NTS is an important cause of foodborne gastroenteritis which is self-limiting ${ }^{[11]}$ and there is no long-term carrier status in NTS like $S$. typhi. ${ }^{[3]}$ Infection with NTS occurs mostly in immunocompromised hosts and among the patients with gastroenteritis, 5\% develop bacteremia who have a tendency to develop focal infections also. ${ }^{[6]}$

There is a high chance of infection with NTS as the age increases, both children and adults are affected. ${ }^{[4]}$ In our study, most of them were in the age group of 20-40 years, and there is no significant increase of infection with increased age. Males are most commonly affected ${ }^{[4]}$ which is similar to our study.

Among the NTS, S. typhimurium and S. enteritidis are the most common human pathogens. ${ }^{[12]}$ In a study from the United States, they found that $S$. typhimurium is the most common NTS isolated accounting for $24 \% .{ }^{[13]}$ The common source of infection includes meat, poultry, and water. ${ }^{[14]}$ In a study from Malawi, they found that S. typhimurium is the most common N TS isolated accounting for $76 \%$ and $75 \%$ in children and adults respectively and $S$. enteritidis accounted for $21 \%$. ${ }^{[15]}$ In our study, $S$. typhimurium was the most common NTS isolated from 15 patients from different samples as mentioned in Table 3 . The risk factors among these patients were SLE on steroids in 6/15 patients, $5 / 15$ patients were cases of pyrexia of unknown origin (PUO) where there was no underlying cause, one patient with malignancy, two patients with cirrhosis of liver, one in the postoperative patient.

In developed countries, like the United States S. enteritidis is one of the common serotypes causing human infections. In 1980, there was outbreaks of S. enteritidis in the United States and they found eggshell as the source of infection. ${ }^{[16,17]}$ In a study from South India, they isolated $71.4 \%$ of $S$. enteritidis from eggshell and egg storing trays. ${ }^{[18]}$ In our study, we isolated four cases of S. enteritidis, one from a patient with acute myeloid leukemia (AML), one from a postoperative patient and two from cases of PUO. As this was a retrospective study, the source of infection of NTS could not be traced in our patients.

We isolated three cases of $S$. lindenberg, two from blood cultures of patients with SLE and one from a patient with chronic kidney disease (CKD). S. lindenberg differs from $S$. typhimurium in having $\mathrm{C} 2$ serogroup $\mathrm{O}$ antigens. ${ }^{[19]}$ To the best of our knowledge and literature search, this is the first report of $S$. lindenberg from blood cultures isolated in our patients.

In a study from Thailand, $S$. weltevreden has been reported as one of the most common causes of human infections. ${ }^{[20]}$ In 1970, it accounted for $<4 \%$ of human infections in India but increased to $29.1 \%$ of human infections in 1972. ${ }^{[21]}$ From India, cases of food poisoning ${ }^{[22]}$ neonatal sepsis, ${ }^{[23]}$ surgical site infection like postcholecystectomy ${ }^{[24]}$ have been reported. In our study, we have isolated three cases of Salmonella weltevreden from blood of SLE patients on steroids.

S. Kentucky was first reported in Egypt during 2002-2005. There was an increase in infections in Europe during 2005-2008, probably acquired through imported food or secondary contamination in the absence of travel to other countries. ${ }^{[25]}$ Although it is rarely causes infection in human, it was found to occur in people travelling to North Africa and South Asia. ${ }^{[26]}$ In our study, we isolated $S$. Kentucky from synovial fluid in a case of ALL with septic arthritis.

Among NTS, S. Cholerasuis causes severe infection in human beings. Though it is rarely reported in the United States and Canada, it ranked $10^{\text {th }}$ among NTS in Thailand during 1988-1993 and is the second common human Salmonellosis causing serotype in Taiwan. ${ }^{[27]}$ It causes bacteremia and may lead to extraintestinal infections without local invasion. ${ }^{[28]}$ The first case of bacteremia from India was from an adult febrile patient in whom S. Cholerasuis was isolated from both blood and urine cultures. ${ }^{[27]}$ In our study, we had one patient with myeloproliferative disorder, and $S$. Cholerasuis was isolated from chest wall abscess from the patient.

NTS causes bacteremia in immunocompromised hosts, and it is considered as a life-threatening condition in adults. In a study from Taiwan, they found 206 patients with NTS bacteremia over a period of 10 years with $36.2 \%$ of patients having an underlying clinical illness. ${ }^{[29]}$ In a study from Bangladesh, they found that NTS accounts for $<0.2 \%$ of all blood cultures. ${ }^{[30]}$ The immune system of the patient helps in defense against NTS; and hence, immunosuppressed status should be checked in a patient with primary NTS bacteremia. ${ }^{[31]}$ In a study from Poland, they found that half of the patients were immunosuppressed from conditions such as SLE, hematological malignancies, renal transplantation, liver cirrhosis, and HIV infection. ${ }^{[32]}$ It also occurs in patients on steroids, malignancy, chronic renal or liver disease, diabetes, or sickle-cell disease, and elderly and newborn patients. ${ }^{[33]}$ We isolated 18/27 of NTS from blood-seven of them were PUO, one patient with CKD, one with cirrhosis, one with postoperative case of mitral valve replacement, one patient with AML on chemotherapy and seven patients with SLE on steroids. Out of 18, 11 patients had an underlying disorder, but in seven patients, the cause was unknown. NTS can cause local infection of any site by hematogeneous spread even though the bacteremia was successfully treated. ${ }^{[6]}$ It can also occur in patients with structural abnormalities 
involving cardiovascular system as valvular heart disease, aneurysms or atherosclerosis, urinary tract, and bony abnormalities. ${ }^{[14]}$ Metastatic spread to any site is common with NTS. Focal infections such as cutaneous abscess, arthritis, peritonitis occurs in $21 \%$ of patients with bacteremia. ${ }^{[34]}$ We isolated NTS one from pleural fluid in a patient with SLE and other from ascitic fluid in a patient with cirrhosis liver. It was isolated from purulent aspirate in two patients (submandibular abscess from a patient with malignancy and chest wall abscess from a patient with myeloproliferative disorder).

Salmonella septic arthritis is estimated to occur at a rate of $0.1 \%-0.2 \% \cdot{ }^{[35]}$ It usually occurs in immunocompromised, but there have been reports in immunocompetent children. ${ }^{[35,36]}$ We isolated three cases of NTS from septic arthritis. All of them were immunosuppressed, two patients with SLE and ALL in one patient.

Infection of urinary tract with NTS occurs only when there is occult urinary tract problem or immunosuppression. ${ }^{[37]}$ We had two cases of NTS isolated from urine one from a case of SLE, and other patient is an immunocompetent adult who had a surgery for fracture of femur.

NTS shows an increase in antimicrobial resistance to the newer antimicrobials posing a serious threat to human population. This occurs mostly due to the widespread use of antimicrobials in food of animals. ${ }^{[38]}$ In a study from China, only $9.2 \%$ of NTS were susceptible to all antibiotics, and S. weltevreden (47\%) and few S. typhimurium (1.5\%) showed pan-susceptible, others showing high rates of resistance ${ }^{[39]}$ In a FoodNet study where they analyzed the incidence of human infections due to $S$. enteritidis they found that they were highly resistant to ampicillin and nalidixic acid. ${ }^{[40]}$ In our study, $5 / 27$ of the isolates were susceptible to nalidixic acid, but others were resistant. Our isolates were susceptible to all other antibiotics indicating that multidrug resistance was not documented among NTS serotypes collected from our medical center as monitored over a period of 3 years.

As it was a retrospective study, our study had certain limitations. The primary source of NTS could not be traced and also patients could not be followed up for the outcome.

\section{Conclusion}

S. typhimurium is the most common serotype isolated in our study. This study highlights the spectrum of NTS causing extraintestinal infections which is an emerging infection occurring mostly in immunosuppressed individuals. Infections due to NTS is often underestimated in the developing countries like India and accounts for the steadily increase in the proportion of human infections during recent decades. The treatment of NTS is becoming difficult with the increasing resistance to antimicrobials. There should be a high degree of clinical suspicion which would help in the early diagnosis and management of patients.

\section{Financial support and sponsorship \\ Nil.}

\section{Conflicts of interest}

There are no conflicts of interest.

\section{References}

1. Sudhaharan S, Padmaja K, Solanki R, Lakshmi V, Umabala P, Aparna B, et al. Extra-intestinal salmonellosis in a tertiary care center in South India. J Infect Dev Ctries 2014;8:831-7.

2. Su LH, Chiu CH. Salmonella: Clinical importance and evolution of nomenclature. Chang Gung Med J 2007;30:210-9.

3. Gal-Mor O, Boyle EC, Grassl GA. Same species, different diseases: How and why typhoidal and non-typhoidal Salmonella enterica serovars differ. Front Microbiol 2014;5:391.

4. Laupland KB, Schønheyder HC, Kennedy KJ, Lyytikäinen O, Valiquette L, Galbraith J, et al. Salmonella enterica bacteraemia: A multi-national population-based cohort study. BMC Infect Dis 2010;10:95.

5. Feasey NA, Dougan G, Kingsley RA, Heyderman RS, Gordon MA. Invasive non-typhoidal Salmonella disease: An emerging and neglected tropical disease in Africa. Lancet 2012;379:2489-99.

6. Hohmann EL. Nontyphoidal salmonellosis. Clin Infect Dis 2001;32:263-9.

7. Kariuki S, Onsare RS. Epidemiology and genomics of invasive nontyphoidal Salmonella infections in Kenya. Clin Infect Dis 2015;61 Suppl 4:S317-24.

8. Shahane V, Muley V, Kagal A, Bharadwaj R. Non-typhoid salmonellosis: Emerging infection in Pune? Indian J Med Microbiol 2007;25:173-4.

9. Oommen S, Nair S, Nair K, Pillai S. Epidemiology of non-typhoidal Salmonella among patients attending a tertiary care centre in central Kerala. J Acad Clin Microbiol 2015;17:12-5.

10. Walsh AL, Phiri AJ, Graham SM, Molyneux EM, Molyneux ME. Bacteremia in febrile Malawian children: Clinical and microbiologic features. Pediatr Infect Dis J 2000;19:312-8.

11. Galanis E, Lo Fo Wong DM, Patrick ME, Binsztein N, Cieslik A, Chalermchikit $\mathrm{T}$, et al. Web-based surveillance and global Salmonella distribution, 2000-2002. Emerg Infect Dis 2006;12:381-8.

12. Saravanan S, Purushothaman V, Murthy TR, Sukumar K, Srinivasan P, Gowthaman V, et al. Molecular epidemiology of nontyphoidal Salmonella in poultry and poultry products in India: Implications for human health. Indian J Microbiol 2015;55:319-26.

13. Olsen SJ, Bishop R, Brenner FW, Roels TH, Bean N, Tauxe RV, et al. The changing epidemiology of Salmonella: Trends in serotypes isolated from humans in the United States, 1987-1997. J Infect Dis 2001;183:753-61.

14. Thong KL, Goh YL, Radu S, Noorzaleha S, Yasin R, Koh YT, et al. Genetic diversity of clinical and environmental strains of Salmonella enterica serotype Weltevreden isolated in Malaysia. J Clin Microbiol 2002;40:2498-503.

15. Gordon MA, Graham SM, Walsh AL, Wilson L, Phiri A, Molyneux E, et al. Epidemics of invasive Salmonella enterica serovar enteritidis and S. Enterica serovar typhimurium infection associated with multidrug resistance among adults and children in Malawi. Clin Infect Dis 2008;46:963-9.

16. Kendall ME, Crim S, Fullerton K, Han PV, Cronquist AB, 
Shiferaw B, et al. Travel-associated enteric infections diagnosed after return to the United States, foodborne diseases active surveillance network (FoodNet), 2004-2009. Clin Infect Dis 2012;54 Suppl 5:S480-7.

17. Patrick ME, Adcock PM, Gomez TM, Altekruse SF, Holland BH, Tauxe RV, et al. Salmonella enteritidis infections, United States, 1985-1999. Emerg Infect Dis 2004;10:1-7.

18. Suresh T, Hatha AA, Sreenivasan D, Sangeetha N, Lashmanaperumalsamy P. Prevalence and antimicrobial resistance of Salmonella enteritidis and other Salmonellas in the eggs and egg-storing trays from retail markets of Coimbatore, South India. Food Microbiol 2006;23:294-9.

19. Alvarez J, Sota M, Vivanco AB, Perales I, Cisterna R, Rementeria A, et al. Development of a multiplex PCR technique for detection and epidemiological typing of Salmonella in human clinical samples. J Clin Microbiol 2004;42:1734-8.

20. Bangtrakulnonth A, Pornreongwong S, Pulsrikarn C, Sawanpanyalert P, Hendriksen RS, Lo Fo Wong DM, et al. Salmonella serovars from humans and other sources in Thailand, 1993-2002. Emerg Infect Dis 2004;10:131-6.

21. Basu S, Sood LR. Salmonella weltevreden: A sero-type of increasing public health importance in India. Trop Geogr Med 1975;27:387-94.

22. Antony B, Dias M, Shetty AK, Rekha B. Food poisoning due to Salmonella enterica serotype Weltevreden in Mangalore. Indian J Med Microbiol 2009;27:257-8.

23. Patil AB, Krishna BV, Chandrasekhar MR. Neonatal sepsis caused by Salmonella enterica serovar Weltevreden. Southeast Asian J Trop Med Public Health 2006;37:1175-8.

24. Ashok R, Anuradha K, Lakshmi V, Kumar A, Bheerappa N, Sastry RA, et al. Postcholecystectomy surgical site infection by Salmonella enterica var. Weltevreden. Surg Infect (Larchmt) 2005;6:449-51.

25. Le Hello S, Hendriksen RS, Doublet B, Fisher I, Nielsen EM, Whichard JM, et al. International spread of an epidemic population of Salmonella enterica serotype Kentucky ST198 resistant to ciprofloxacin. J Infect Dis 2011;204:675-84.

26. Tasmin R, Hasan NA, Grim CJ, Grant A, Choi SY, Alam MS, et al. Genotypic and phenotypic characterization of multidrug resistant Salmonella typhimurium and Salmonella Kentucky strains recovered from chicken carcasses. PLoS One 2017;12:e0176938.

27. Chiu $\mathrm{CH}, \mathrm{Su} \mathrm{LH}, \mathrm{Chu}$ C. Salmonella enterica serotype choleraesuis: Epidemiology, pathogenesis, clinical disease, and treatment. Clin Microbiol Rev 2004;17:311-22.

28. Chen PL, Wu CJ, Chang CM, Lee HC, Lee NY, Shih HI, et al. Extraintestinal focal infections in adults with Salmonella enterica Serotype Choleraesuis bacteremia. J Microbiol Immunol Infect 2007;40:240-7.

29. Li CW, Chen PL, Lee NY, Lee HC, Chang CM, Lee CC, et al. Non-typhoidal Salmonella bacteremia among adults: An adverse prognosis in patients with malignancy. J Microbiol Immunol Infect 2012; 45:343-9.

30. Shahunja KM, Leung DT, Ahmed T, Bardhan PK, Ahmed D, Qadri F, et al. Factors associated with non-typhoidal Salmonella bacteremia versus typhoidal Salmonella bacteremia in patients presenting for care in an urban diarrheal disease hospital in Bangladesh. PLoS Negl Trop Dis 2015;9:e0004066.

31. Brown M, Eykyn SJ. Non-typhoidal Salmonella bacteraemia without gastroenteritis: A marker of underlying immunosuppression. Review of cases at St. Thomas' hospital 1970-1999. J Infect 2000;41:256-9.

32. Kedzierska J, Piatkowska-Jakubas B, Kedzierska A, Biesiada G, Brzychczy A, Parnicka A, et al. Clinical presentation of extraintestinal infections caused by non-typhoid Salmonella serotypes among patients at the university hospital in Cracow during an 7-year period. Pol J Microbiol 2008;57:41-7.

33. Gordon MA. Salmonella infections in immunocompromised adults. J Infect 2008;56:413-22.

34. Chen YH, Chen TP, Lu PL, Su YC, Hwang KP, Tsai JJ, et al. Salmonella choleraesuis bacteremia in Southern Taiwan. Kaohsiung J Med Sci 1999;15:202-8.

35. Lee SC, Yang PH, Shieh WB, Lasserre R. Bacteremia due to non-typhi Salmonella: Analysis of 64 cases and review. Clin Infect Dis 1994;19:693-6.

36. Lim LE, Dowdle B, Gardiner D. Nontyphoidal Salmonella septic arthritis in an immunocompetent child - An atypical presentation highlighting the importance of definitive diagnosis. Infect Dis Health 2017;22:89-91.

37. Gagnon J, Labbé R, Laroche B. Salmonella urinary tract infection: A vascular emergency. Can J Surg 2007;50:221-2.

38. Su LH, Chiu $\mathrm{CH}, \mathrm{Chu} \mathrm{C}$, Ou JT. Antimicrobial resistance in nontyphoid Salmonella serotypes: A global challenge. Clin Infect Dis 2004;39:546-51.

39. Liang Z, Ke B, Deng X, Liang J, Ran L, Lu L, et al. Serotypes, seasonal trends, and antibiotic resistance of non-typhoidal Salmonella from human patients in Guangdong Province, China, 2009-2012. BMC Infect Dis 2015;15:53.

40. Chai SJ, White PL, Lathrop SL, Solghan SM, Medus C, McGlinchey BM, et al. Salmonella enterica serotype enteritidis: Increasing incidence of domestically acquired infections. Clin Infect Dis 2012;54 Suppl 5:S488-97. 